\title{
BOAS PRÁTICAS ASSITENCIAIS DIRECIONADAS À GESTANTE EM TEMPOS DE COVID-19
}

\author{
GOOD ASSISTANCE PRACTICES DIRECTED TO PREGNANT \\ WOMEN INCOVID-19 TIMES
}

\author{
Anny Isabelly Medeiros de Góes ${ }^{1}$ \\ Renata Ferreira de Araújo ${ }^{2}$ \\ Karén Kelyany Duarte Costa ${ }^{3}$ \\ Inácia Sátiro Xavier de França ${ }^{4}$
}

\begin{abstract}
RESUMO: Objetivo: Identificar as evidências cientificas sobre os cuidados à gestante no contexto da pandemia do COVID-19. Método: Revisão integrativa da literatura realizada no mês de fevereiro de 2021, nas bases de dados Cumulative Index to Nursing and Allied Health Literature (CINAHL), Scientific Electronic Library Online (SciELO), Literatura Latino-Americana e do Caribe em Ciências da Saúde (LILACS), Medical Literature Analysis and Retrieval System Online (MEDLINE) e SCOPUS por meio dos descritores "Coronavirus", "Pregnant Women" e "Nursing Care". Foram utilizados como critérios de inclusão artigos completos, em português, inglês e espanhol com publicações no último ano. Resultados: Oito estudos compuseram a amostra final, dos quais desvelou-se duas categorias analíticas: (1)Cuidados de Enfermagem direcionados as gestantes com covid-19 e (2)Características clínicas importantes para tomadas de decisões do cuidado às gestantes com covid-19. Conclusão: Evidencia-se a transcendência do papel da enfermagem na prática assistencial, educativa e gerencial para colaborar na adesão e realização às boas práticas recomendadas em âmbito nacional e internacional, considerando-se a realidade local e a necessidade de atualização constante na temática.
\end{abstract}

Palavras chave: Coronavírus. Gestantes. Enfermagem. Cuidados de Enfermagem.

\footnotetext{
${ }^{1}$ Graduanda em Enfermagem pela Universidade Estadual da Paraíba e Bolsista de Iniciação Científica, UEPB.

${ }^{2}$ Graduanda em Enfermagem pela Universidade Estadual da Paraíba e Bolsista de Iniciação Científica, CNPq,UEPB.

${ }^{3}$ Graduanda em Enfermagem pela Universidade Estadual da Paraíba.

${ }^{4}$ Doutora em Enfermagem pela Universidade Federal do Ceará.
} 
ABSTRACT: Objective: To identify scientific evidence on care for pregnant women in the context of the COVID-19 pandemic. Method: Integrative literature review conducted in February 2021, in the databases Cumulative Index to Nursing and Allied Health Literature (CINAHL), Scientific Electronic Library Online (SciELO), Latin American and Caribbean Literature in Health Sciences (LILACS), Medical Literature Analysis and Retrieval System Online (MEDLINE) and SCOPUS through the descriptors "Coronavirus", "Pregnant Women" and "Nursing Care". Full articles in Portuguese, English and Spanish with publications in the last year were used as inclusion criteria. Results: Eight studies made up the final sample, of which two analytical categories were unveiled: (1)- Nursing care directed to pregnant women with covid-19 and (2)-Important clinical characteristics for decision-making care for pregnant women with covid-19. Conclusion: The role of nursing in health care, educational and managerial practice is highlighted to collaborate in adhering to and implementing the best practices recommended at national and international levels, considering the local reality and the need for constant updatingof the theme.

Keywords: Coronavirus. Pregnant Women. Nurse. Nursing Care. 


\section{INTRODUÇÃO}

As infecções emergentes, a exemplo da gripe causada pelo $\mathrm{H} 1 \mathrm{~N} 1$ e o vírus da Zika, mostraram ter um importante acometimento em mulheres grávidas e em seus fetos, dispondo-as a um maior risco de complicações (RASMUSSEN et al., 2011). As mulheres grávidas tornam-se mais vulneráveis ao adoecimento devido a alterações imunofisiológicas que ocorrem durante a gravidez. O surgimento de um coronavírus até então não visto em humanos, relatadopela primeira vez em Wuhan, China em dezembro de 2019 despertou o interesse de todo o mundo pela sua alta capacidade de contágio e adoecimento (SISTON et al., 2010; MOORE et al., 2017).

O coronavírus pode causar uma variedade de casos que se manifestam desde um resfriado leve até um grave problema respiratório, como a pneumonia. Em casos leves a sintomatologia é apresentada por febre, tosse, cefaleia e diarreia e nos casos mais graves pode apresentar linfopenia, leucopenia e trombocitopenia. A doença foi reconhecida pela Organização Mundial de Saúde como uma emergência global de saúde pública. (RASMUSSEN et al., 2020; PEERI et al., 2020; LI et al., 2020).

A infecção por SARS durante a gravidez apresenta efeitos adversos maternos e neonatais, incluindo aborto espontâneo, parto prematuro, restrição de crescimento intrauterino, intubação traqueal, hospitalização e coagulação intravascular (RASMUSSEN et al., 2020).

Destaca-se a importância da realização de um cuidado efetivo à gestante, visando a redução de agravos gestacionais e neonatais. Assim sendo, é pertinente que os enfermeiros desenvolvam práticas assistenciais e orientações atualizadas de modo a garantir os cuidados necessários de forma segura e efetiva.

Portanto, faz-se necessário mapear as melhores evidencias sobre a temática que subsidiem as ações assistências, educativas e gerenciais dos enfermeiros e suas equipes durante e após o contexto da pandemia causada pelo novo coronavírus. 
Destarte, o objetivo do estudo consistiu em identificar as evidências cientificas sobre os cuidados à gestante no contexto dapandemia do COVID-19.

\section{MÉTODO}

Revisão integrativa da literatura desenvolvida através das seis etapas propostas por MENDES et al., 2008. A questão norteadora da pesquisa foi elaborada por meio da estratégia Population, Intervention, Comparison, Outcome (PICO), utilizada na Prática Baseada em Evidências (PBE) (SANTOS et al., 2007). Sendo assim, P- gestantes; I- cuidados de enfermagem; e Co- COVID-19. Logo, a questão norteadora foi: Quais as evidências cientificas centradas nos cuidados à gestante em tempo de COVID-19?

Para a busca dos artigos, utilizou-se das bases de dados: Cumulative Index to Nursing and Allied Health Literature (CINAHL), Scientific Electronic Library Online (SciELO), Literatura Latino-Americana e do Caribe em Ciências da Saúde (LILACS), Medical Literature Analysis and Retrieval System Online (MEDLINE) e SCOPUS. Os termos controlados foram utilizados em inglês, por meio do operador booleano "AND", sendo eles: "Coronavirus", "Pregnant Women" e "Nursing Care". Com base nas associações dos termos, iniciou-se uma leitura flutuante dos títulos e resumos dos estudos encontrados para uma seleção prévia. A produção dos dados ocorreu no final de fevereiro de 2021.

Para os critérios de inclusão tem-se: artigos completos disponíveis eletronicamente nos idiomas português, inglês e espanhol, sendo do último ano (2020-2021) e que respondam à temática do estudo. Como critérios de exclusão estão as publicações duplicadas e que correspondem a literatura cinzenta.

Os níveis de evidência foram classificados através da proposta de MELNYK E FINEOUT-OVERHOLT, 2005, onde: I para revisões sistemáticas e meta-análise de ensaios clínicos randomizados; II para ensaios clínicos randomizados; III para ensaio controlado não randomizado; IV para estudos caso-controle ou coorte; V para revisões sistemáticas de estudos qualitativos ou descritivos; VI para estudos 
qualitativos ou descritivos; e VII para parecer de autoridades e/ou relatórios de comitês de especialistas.

\section{RESULTADOS}

A partir dos cruzamentos obteve-se um universo de 27 títulos e resumos lidos, a partir dos quais 15 publicações foram identificadas inicialmente pela aproximação com o foco do estudo, como consta no Quadro 1.

Em seguida, com a exclusão de 4 publicações duplicadas devido ao critério de exclusão previamente estabelecido, restaram 11 manuscritos que foram lidos na íntegra. Destes, 8 estudos constituíram a amostra final. Para a seleção dos estudos, utilizou-se das recomendações fornecidas pelo Preferred Reporting Items for Systematic Reviews and Meta-Analyses (PRISMA). O PRISMA é composto de um checklist com 27 itens e um diagrama de fluxo para seleção das publicações (GALVÃO; PANSANI; HARRAD, 2015), apresentado na Figura 1. 
Quadro 1 - Estratégias de busca e número de manuscritos obtidos nos recursos informacionais. Campina Grande, PB, Brasil, 2021. ( $N=15)$.

\begin{tabular}{|c|c|c|c|}
\hline Base de Dados & Estratégia de Busca & $\begin{array}{l}\text { Localizados } \\
\text { nas buscas }\end{array}$ & $\begin{array}{l}\text { Identificados } \\
\text { por título e } \\
\text { resumo }\end{array}$ \\
\hline CINAHL & $\begin{array}{l}\text { Coronavirus [MeSH Terms] AND Pregnant Women } \\
{[\text { [MeSH Terms] AND Nursing Care [MeSH Terms] }} \\
{[\text { Title/ Abstract/ Full text] }}\end{array}$ & 1 & 1 \\
\hline LILACS & $\begin{array}{l}\text { Coronavirus [MeSH Terms] AND Pregnant Women } \\
\text { [MeSH Terms] AND Nursing Care [MeSH Terms] } \\
{[\text { [Title/Abstract/ Full text] }}\end{array}$ & 1 & 1 \\
\hline MEDLINE & $\begin{array}{l}\text { Coronavirus [MeSH Terms] AND Pregnant Women } \\
{[\text { [MeSH Terms] AND Nursing Care [MeSH Terms] }} \\
{[\text { [Title/Abstract/ Full text] }}\end{array}$ & 9 & 4 \\
\hline SCIELO & $\begin{array}{l}\text { Coronavirus [MeSH Terms] AND Pregnant Women } \\
{[\text { [MeSH Terms] AND Nursing Care [MeSH Terms] }} \\
{[\text { [Title/ Abstract/ Full text] }}\end{array}$ & 2 & 2 \\
\hline SCOPUS & $\begin{array}{l}\text { Coronavirus [MeSH Terms] AND Pregnant Women } \\
\text { [MeSH Terms] AND Nursing Care [MeSH Terms] } \\
{[\text { [Title/Abstract/ Full text] }}\end{array}$ & 14 & 7 \\
\hline TOTAL & & 27 & 15 \\
\hline
\end{tabular}


Figura 1: Fluxograma do processo de identificação, seleção e inclusão dos estudos. Adaptaçãoda Recomendação PRISMA. Campina Grande, PB, Brasil, 2021.
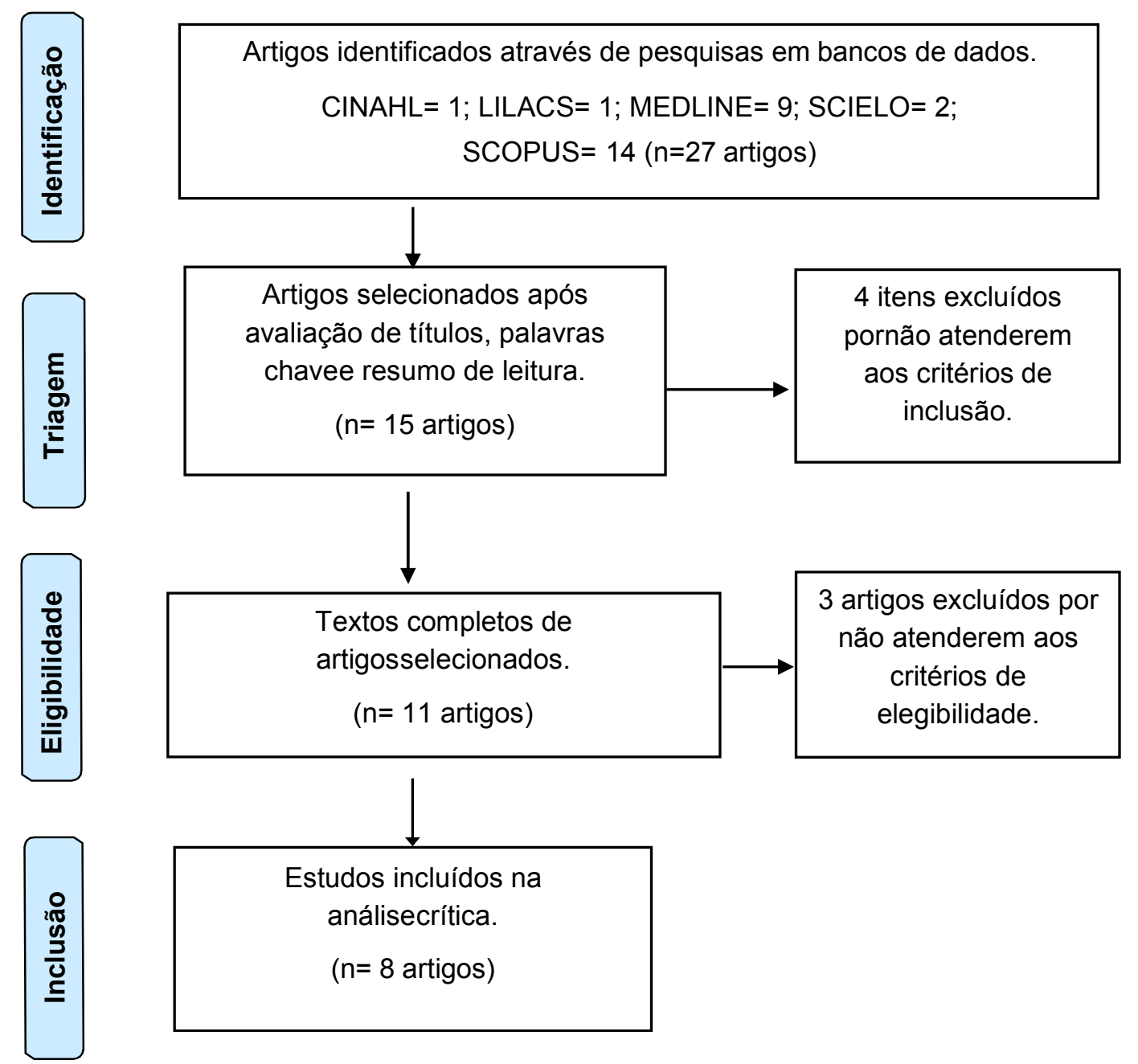
Quadro 2- Síntese dos principais dados extraídos dos artigos selecionados nas bases de dados.Campina Grade, PB, Brasil, 2021.

\begin{tabular}{|c|c|c|c|c|c|c|}
\hline Identificação & Título & $\begin{array}{l}\text { País I } \\
\text { Ano }\end{array}$ & Objetivo & $\begin{array}{l}\text { Tipo de } \\
\text { estudo }\end{array}$ & $\begin{array}{c}\text { Nível de } \\
\text { Evidência }\end{array}$ & Categoria \\
\hline 1. & $\begin{array}{c}\text { COVID-19 e a } \\
\text { produção de } \\
\text { conhecimento sobre } \\
\text { as recomendaçõe s } \\
\text { na gravidez:revisão } \\
\text { deescopo }\end{array}$ & $\begin{array}{l}\text { Brasil, } \\
2020\end{array}$ & \begin{tabular}{|} 
Mapear a produção \\
deconhecimento \\
sobre as \\
recomendaçõespara \\
a assistência à \\
gestante no \\
enfrentamento do \\
novo Coronavírus.
\end{tabular} & $\begin{array}{l}\text { Revisão de } \\
\text { escopo }\end{array}$ & V & $\begin{array}{c}\text { Cuidados } \\
\text { direcionados } \\
\text { (1) }\end{array}$ \\
\hline 2. & $\begin{array}{c}\text { Evaluating clinical } \\
\text { courseand risk factors } \\
\text { of infectionand } \\
\text { demographic } \\
\text { characteristics of } \\
\text { pregnantwomen with } \\
\text { COVID-19 in } \\
\text { Hamadan Province, } \\
\text { West of Iran } \\
\end{array}$ & $\begin{array}{l}\text { Irã, } \\
2020\end{array}$ & \begin{tabular}{|c|} 
Avaliar o curso \\
clínico e osfatores \\
de riscode mulheres \\
grávidas com \\
diagnóstico de \\
COVID 19 na \\
província de \\
Hamadan, oeste do \\
Irã \\
\end{tabular} & $\begin{array}{l}\text { Estudo de } \\
\text { coorte } \\
\text { retrospectivo }\end{array}$ & IV & $\begin{array}{l}\text { Característica } \\
\text { sclínicas (2) }\end{array}$ \\
\hline 3. & $\begin{array}{c}\text { Pregnancy outcomes } \\
\text { among symptomatic } \\
\text { and asymptomatic } \\
\text { women infected with } \\
\text { COVID-19 in the west } \\
\text { oflran: a case-control } \\
\text { study }\end{array}$ & $\begin{array}{l}\text { Irã, } \\
2020\end{array}$ & \begin{tabular}{|c|} 
Investigar os \\
resultados da \\
gravidez entre \\
mulheres \\
sintomáticas e \\
assintomáticas \\
infectadas com \\
doença coronavírus \\
2019 (COVID-19) \\
no oeste do Irã \\
\end{tabular} & $\begin{array}{l}\text { Estudo de } \\
\text { caso - } \\
\text { controle }\end{array}$ & IV & $\begin{array}{l}\text { Característica } \\
\text { sclínicas (2) }\end{array}$ \\
\hline 4. & $\begin{array}{l}\text { Improving thequality } \\
\text { of carein } \\
\text { pregnancyand } \\
\text { childbirthwith } \\
\text { coronavirus (COVID- } \\
\text { 19): a systematic } \\
\text { review }\end{array}$ & $\begin{array}{l}\text { Irã, } \\
2020\end{array}$ & \begin{tabular}{|} 
Este estudo foi \\
desenhado para \\
melhorar a \\
qualidade do \\
atendimento pelos \\
profissionais de \\
saúde pormeio do \\
que eles precisam \\
saber sobre o \\
coronavírus durante \\
agravidez e o parto.
\end{tabular} & $\begin{array}{c}\text { Revisão } \\
\text { Sistemática }\end{array}$ & V & $\begin{array}{c}\text { Cuidados } \\
\text { direcionados } \\
\text { (1) }\end{array}$ \\
\hline
\end{tabular}




\begin{tabular}{|c|c|c|c|c|c|c|}
\hline 5. & \begin{tabular}{|} 
Patients With COVID- \\
19 Undergoing \\
Cesarean Deliveries: \\
Adapting theOR Suite \\
and Perioperative \\
Care to Prevent \\
Transmission
\end{tabular} & $\begin{array}{c}\text { Wuhan, } \\
2020\end{array}$ & \begin{tabular}{|c|} 
Analisar \\
retrospectivamente \\
o processode \\
enfermagem \\
perioperatório, \\
incluindo \\
preparação dasala \\
de cirurgia, \\
cuidados \\
intraoperatórios e \\
limpeza dasala de \\
cirurgia, para \\
mulheres com \\
COVID - 19 \\
Submetidas apartos \\
cesáreos.
\end{tabular} & $\begin{array}{l}\text { Estudo } \\
\text { Descritivo }\end{array}$ & VI & $\begin{array}{c}\text { Cuidados } \\
\text { direcionados } \\
\text { (1) }\end{array}$ \\
\hline 6. & $\begin{array}{c}\text { Management of } \\
\text { pregnantlaboring } \\
\text { women duringCOVID- } \\
19 \text { pandemic }\end{array}$ & $\begin{array}{l}\text { India, } \\
2020\end{array}$ & $\begin{array}{c}\text { Contribuir } \\
\text { efetivamente para a } \\
\text { gestãode } \\
\text { parturientesdurante } \\
\text { a pandemia do } \\
\text { COVID-19. } \\
\end{array}$ & $\begin{array}{l}\text { Estudo } \\
\text { Descritivo }\end{array}$ & VI & $\begin{array}{c}\text { Cuidados } \\
\text { direcionados } \\
\text { (1) }\end{array}$ \\
\hline 7. & $\begin{array}{c}\text { Characteristic and } \\
\text { short-termobstetric } \\
\text { outcomes in acase } \\
\text { series of } 67 \text { women } \\
\text { test-positive forSARS- } \\
\text { CoV-2 in Stockholm, } \\
\text { Sweden }\end{array}$ & $\begin{array}{c}\text { Suécia, } \\
2020\end{array}$ & \begin{tabular}{|c|} 
Descrever as \\
características \\
clínicas demulheres \\
positivas para o \\
SARS-CoV-2 e \\
seus recém- \\
nascidos, relatando \\
os resultados \\
maternos e \\
neonatais decurto \\
prazo.
\end{tabular} & $\begin{array}{l}\text { Estudo } \\
\text { Descritivo }\end{array}$ & VI & $\begin{array}{c}\text { Característica } \\
\text { sclínicas (2) }\end{array}$ \\
\hline 8. & $\begin{array}{c}\text { The Psychological } \\
\text { Experience of } \\
\text { Obstetric Patients and } \\
\text { Health CareWorkers } \\
\text { afterlmplementatio n } \\
\text { of UniversalSARS- } \\
\text { CoV-2 Testing }\end{array}$ & $\begin{array}{l}\text { Pensilv } \\
\text { ânia, } \\
2020\end{array}$ & \begin{tabular}{|c|} 
Referir a \\
hospitalização,o \\
pós-parto \\
precoce e a \\
experiência \\
psicológica de \\
pacientes \\
obstétricas \\
assintomáticas \\
testadas para \\
quadros agudos \\
gravesda síndrome \\
respiratória causada \\
pelo coronavírus.
\end{tabular} & $\begin{array}{l}\text { Estudo de } \\
\text { coorte } \\
\text { retrospectivo }\end{array}$ & IV & $\begin{array}{l}\text { Característica } \\
\text { sclínicas (2) }\end{array}$ \\
\hline
\end{tabular}

Fontes: Dados da pesquisa, 2021.

Mediante a análise crítica dos artigos que compuseram a amostra, desvelouse duas categorias: (1) - Cuidados de Enfermagem direcionados as gestantes com covid-19 e (2) - Características clínicas importantes para tomadas de decisões do 
cuidado às gestantes com covid-19. Os artigos identificados em 1, 4, 5 e 6 são categorizados em cuidados direcionados e2,3, 7 e 8 enquadram-se na categoria das características clínicas da doença.

\section{DISCUSSÃO}

$\mathrm{Na}$ prática assistencial para as mulheres gestantes no contexto da pandemia do covid-19, engloba um cenário atual e desconhecido, onde são necessárias evidências cientificas para uma prática segura e benéfica as pacientes. Diante de todo exposto, é de relevância que os enfermeiros, conheçam a sintomatologia, curso clínico e complicações do novo coronavírus com o objetivo de prevenir o agravo dessa enfermidade, intervindo antecipadamente por meio de orientações e encaminhamentos necessários para gestante e feto (ESTRELA, 2020).

O curso clínico da covid-19 nas gestantes desvela-se com os sintomas mais comuns: febre, tosse e falta de ar. Nos exames de imagens de tórax apresentam múltiplas manchas e opacidade em vidro fosco. Além dos sintomas, as mulheres apresentaram uma maior vulnerabilidade para a via de parto cesariana e os seus bebês apresentaram maiores índices de prematuridade (SATTARI, 2020). Diante disso, é imprescindível que a Enfermagem possua conhecimento do percurso da doença e as necessidades de intervenções para uma assistência qualificada por meio da Sistematização de Enfermagem (SAE).

A chance de parto cesariano em mulheres sintomáticas é de quatro vezes maior em relação as mulheres assintomáticas, além do mais, os bebês ao nascerem apresentaram baixo peso em proporção significativa. Deste modo, é importante a recomendação do teste $\mathrm{PCR}$ (SWAB) para todas as gestantes na admissão para o parto, porém é uma realidade ainda inacessível no Brasil (JENABI, 2020).

A prioridade de assistência deve ser centrada na melhoria do atendimento da gestante durante a gravidez e parto, propondo conhecimentos para as mesmas sobre o vírus, capacitação profissional para lidar com as situações envolvendo covid-19, gestantes e o neonatais. Assim, o compartilhamento dos dados das pesquisas ajuda 
para que os países afetados se respaldem em conhecimentos já existentes, no sentido de fundamentar-se na construção de protocolos de atendimentos e prevenir complicações (ABDOLLAHPOUR, 2020).

No hospital Tongji em Wuhan, na China estabeleceu-se as seguintes medidas como protocolos: criação de isolamento para mulheres gestantes com covid-19, após a cesariana desligar o ar como um método para conter partículas de ar potencialmente infecciosas, visandominimizar a chance de contaminação na sala de cirurgia, além da desinfecção de materiais utilizados e de contato com a mulher infectada (ZOU, 2020).

Para prevenção de contágio do recém-nascido, havia a separação do recémnascido e da mãe, evitando contato pele a pele e hora de ouro. No momento do parto, realizava-se o clampeamento e corte no cordão umbilical o mais rápido possível. Além disso, os 6 bebês que nasceram nesse hospital por covid-19, ficaram por 14 dias isolados de suas mães após o parto, interferindo negativamente na amamentação e no vínculo afetivo (ZOU, 2020).

Os cuidados com as pacientes grávidas acometidas com a covid-19, as quais se submeterem a parto cesariano em Wuhan, resultou em mudanças na sala de cirurgia e nos cuidados perioperatórios para uma sistematização segura para equipe e outros pacientes. Assim, compreendemos a importância de novas estratégias para inibir a propagação do vírus, visando a segurança nos procedimentos invasivos do binômino mãe e filho (ZOU, 2020).

\section{CONCLUSÃO}

Evidencia-se a transcendência do papel da enfermagem na prática assistencial, educativa e gerencial para colaborar na adesão e realização às boas práticas recomendadas em âmbito nacional e internacional, considerando-se a realidade local e a necessidade de atualização constante na temática. São necessárias as produções de novas pesquisas, que contribuam para a formulação de diretrizes assistenciais aos profissionais de saúde de modo a contribuir para a redução da 
morbimortalidade gestacional e neonatal causada pelo novo coronavírus, fornecendo às gestantes um gestar e parir saudável e harmonioso durante e pós-pandemia.

\section{REFERÊNCIAS BIBLIOGRÁFICAS}

ABDOLLAHPOUR, S.; KHADIVZADEH, T. Improving the quality of care in pregnancy and childbirth with coronavirus (COVID-19): a systematic review. The Journal of Maternal- Fetal \& Neonatal Medicine, p. 1-9, 2020.

BENDER, W. R.; SRINIVAS, S.; COUTIFARIS, P. et al. The Psychological Experience of Obstetric Patients and Health Care Workers after Implementation of Universal SARS-CoV-2 Testing. American Journal of Perinatology. v. 37, n. 12, p. 1271-1279, 2020.

ESTRELA, F. M. et al. Gestantes no contexto da pandemia da Covid-19: reflexões e desafios. Physis: Revista de Saúde Coletiva, v. 30, n. 2, 2020.

GALVÃO, T. F.; PANSANI, T.S.; HARRAD, D. Reporting Items for Systematic Reviews andMetaAnalyses: The PRISMA Statement. Epidemiologia e Serviços de Saúde. v. 24, p. 335- 42, 2015.

JAIN, K.; BATHIA, N.; GREWAL, A.; PANDYA, S. T.; et al. Management of pregnant laboring women during COVID-19 pandemic. Journal of Anaesthesiology Clinical Pharmacology. v. 36, n. 1, 2020.

JENABI, E. et al. Pregnancy outcomes among symptomatic and asymptomatic women infected with COVID-19 in the west of Iran: a case-control study. The Journal of Maternal-Fetal \& Neonatal Medicine, p. 1-3, 2020.

LI, X.; GENG, M.; PENG, Y.; MENG, L.; LU, S. Molecular immune pathogenesis and diagnosis of COVID-19. J Pharm Anal. v. 10, n. 2, p. 102-8, 2020.

MASCARENHAS, V. H. A. et al. COVID-19 and the production of knowledge regarding recommendations during pregnancy: a scoping review. Revista latino-americana de enfermagem, v. 28, 2020.

MENDES, K. D. S.; SILVEIRA, R. C. C. P.; GALVÃO, C. M. Revisão Integrativa: Método de Pesquisa para a Incorporação de Evidências na Saúde e na Enfermagem. Texto e Contexto Enfermagem. v.17, n.4, pp.758-764. Florianópolis, 2008.

MELNYK, B. M.; FINEOUT-OVERHOLT, E. Making the case for evidence-based practice. In: Melnyk BM, Fineout-Overholt E. Evidence-based practice in nursing \& healthcare. A guide to best practice. Philadelphia: Lippincot Williams \& Wilkins, p.3-24, 2005.

MOORE, C. A.; STAPLES, J. E.; DOBYNS, W. B. et al. Characterizing the Pattern of Anomalies in Congenital Zika Syndrome for Pediatric Clinicians. JAMA Pediatrics. v. 171, n.3, p. 288-295, 2017.

PEERI, N.C.; SHRESTHA, N.; RAHMAN, M.S; ZAKI, R.; TAN, Z.; BIBI, S. et al. The SARS, MERS and novel coronavirus (COVID-19) epidemics, the newest and biggest global health threats: what leassons have we learned? In J Epidemiol. p. 1-10, 2020.

PETERS, M. D. J.; GODFREY, C. M.; KHALIL, H. et al. Guidance for conducting systematic scoping reviews. International Journal of Evidence-Based Healthcare. University of Adelaide, Joanna Briggs Institute, 2015. 
RASMUSSEN, S. A.; HAYES E. B. Public Health Approach to Emerging Infections Among Pregnant Women. American Journal of Public Health, 2011.

RASMUSSEN, S. A.; SMULIAN, J. C.; LEDNICKY, J. A.; WEN, T.S.; JAMIESON, D. J. Coronavirus Disease 2019 (COVID-19) and Pregnancy: What obstetricians need to know. Am J Obstet Gynecol. v. 222, n. 5, p.415-26, 2020.

REMAEUS, K.; SAVCHENKO, J.; WENDEL, S. B. et al. Characteristics and short-term obstetric outcomes in a case series of 67 women test-positive for SARS-CoV-2 in Stockholm, Sweden. Acta Obstet Gynecol Scand. v.99, p. 1626-1631, 2020.

SANTOS, C. M.; PIMENTA, C. B.; NOBRE, M. R. The PICO stategy for the research question construction and evidence search. Rev Latino Americana de Enfermagem. v. 15, p. 508-11, 2007.

SATTARI, M. et al. Evaluating clinical course and risk factors of infection and demographic characteristics of pregnant women with COVID-19 in Hamadan Province, West of Iran. Journal of research in health sciences, v. 20, n. 3, p. 488, 2020.

SISTON, A. M.; RASMUSSEN, S. A.; HONEIN, M. A. et al. Pandemic 2009 Influenza A (H1N1) Virus Illness Among Pregnant Women in the United States. JAMA. v. 303, n. 15, p. 1517-1525, 2010.

ZOU, K.; CHEN, H.; LIU, Y. Patients With COVID-19 Undergoing Cesarean Deliveries: Adapting the OR Suite and Perioperative Care to Prevent Transmission. AORN journal, v. 112, n. 3, p. 217-224, 2020. 